\title{
Orbital apex syndrome after trauma in a 6-year-old - a rare occurrence
}

\author{
A Ahmed, MB BCh, Dip Ophth (SA) \\ Registrar, Department of Ophthalmology, Grey's Hospital, Pietermaritzburg, and School of Clinical Medicine, College of Health Sciences, \\ Nelson R Mandela School of Medicine, University of KwaZulu-Natal, Durban, South Africa
}

Corresponding author: A Ahmed (afrozeahmed242@gmail.com)

\begin{abstract}
Orbital apex syndrome is rare, but can occur as a consequence of trauma from fracture of the medial orbit. This case report highlights the fact that a high index of suspicion is needed when a patient presents with a facial injury, especially in children who cannot give an account of the actual events that transpired. Radiological investigation should be done early when an underlying injury is suspected in a trauma patient. A low threshold for computed tomography should be maintained when proptosis and vision loss are present.
\end{abstract}

S Afr Med J 2017;107(9):747-749. DOI:10.7196/SAMJ.2017.v107i9.12465

\section{Case report}

A 6-year-old boy was brought to the casualty department at Northdale Hospital, Pietermaritzburg, South Africa, after having fallen onto a tree stump while playing in a park. There was no history of loss of consciousness, vomiting or seizures after the fall. He was awake and orientated to person, place and time when he arrived at the hospital. On examination he was found to have swelling and abrasions on the middle of the forehead, the left cheek and the left upper eyelid. There were no eyelid lacerations. The pupils were equal and reactive. There was no documented hyphaema or chemosis of the eye, but there was conjunctivitis. There was no objective documentation or examination of the eye movements. A skull radiograph was done, and no fractures were noted. The final assessment of the patient by the casualty officer was blunt-force trauma with no vision loss, and he was discharged with analgesics and chloromycetin ointment.

Nine days later the patient was brought to casualty again, complaining of headaches and pain originating from the left eye. There was also an unusual complaint of bleeding from the eye. According to the notes, his vital signs were documented as stable. On examination of the eye, there was chemosis but no hyphaema or conjunctivitis. All eye movements were documented as having a full range in all directions. The eyes were irrigated, and the patient was discharged again with chloromycetin ointment and analgesics. Of note, this was a different doctor from the first, and no further radiological investigations were done.

Three days later the patient's mother brought him to Northdale Hospital for the third time, and he was finally referred to the eye clinic. In the eye clinic his visual acuity was assessed as 6/18 in the right eye and poor light perception in the left eye. The intraocular pressure (IOP) in the right eye was $22 \mathrm{mmHg}$ and that the left eye $33 \mathrm{mmHg}$. There was a $4 \mathrm{~mm}$ proptosis of the left eye compared with the right eye, measured with an exophthalmometer. The findings on anterior segment examination of the right eye were normal. Examination of the fundus showed a normal pink disc with a $0.1 \mathrm{cup} /$ disc ratio. The macula of both eyes was normal.

Examination of the left eye showed the following:

- There was complete ptosis of the left eyelid.

- Proptosis was evident.

- The conjunctiva was clear.
- The cornea was clear.

- The anterior chamber was deep and no cells or flare were noted.

- The lens was clear, but the pupil was mid-dilated.

- There was a reactive afferent pupillary defect.

- The extraocular muscles showed no movement in all cardinal directions of the eye, and it was centrally fixed in the orbit.

- Fundus examination showed a pink disc, a cup/disc ratio of 0.2 , and no swelling of the optic disc.

The patient was assessed as having complete ophthalmoplegia, ptosis and proptosis of the left eye. He was immediately referred to a tertiary hospital and an emergency computed tomography (CT) scan of the brain and orbits with contrast was booked for the same day. The scan revealed a left medial blow-out fracture through the anterior lamina papyracea with no extraocular muscle entrapment. There was a haematoma of the left extraocular muscles and enlargement of the left optic nerve. A small haematoma extended through to the cavernous sinus. All the paranasal sinuses were normal. Further details from the CT report are given below.

Intravenous dexamethasone, analgesia and antibiotics were prescribed. There was no improvement over the following 3 days in the ward, and the patient was discharged with a review date. Unfortunately owing to the delay in diagnosis the eye was permanently damaged and the outcome was poor.

\section{Further details from the $\mathrm{CT}$ report}

- Orbits. There was enhancement and thickening of the left inferior oblique muscle measuring $8.31 \mathrm{~mm}$. The right inferior oblique muscle measured $3.02 \mathrm{~mm}$. It caused indentation of the globe inferomedially, and lateral displacement of the globe. The left medial rectus muscle was thickened (left medial rectus $6.48 \mathrm{~mm}$, right medial rectus $3.66 \mathrm{~mm}$ ). Retrobulbar intraconal fat stranding was present. There was no evidence of vitreous haemorrhage. The globe was intact. The left optic nerve was enlarged (left optic nerve $7.37 \mathrm{~mm}$, right optic nerve $6.16 \mathrm{~mm}$ ). There was a blowout fracture of the anterior lamina papyracea with extrusion of fat but no extraocular muscle entrapment. No orbital emphysema was seen. Sellar and suprasellar structures were normal. Fig. 1 shows the fracture, the intraconal fat stranding and the enlarged optic nerve. 


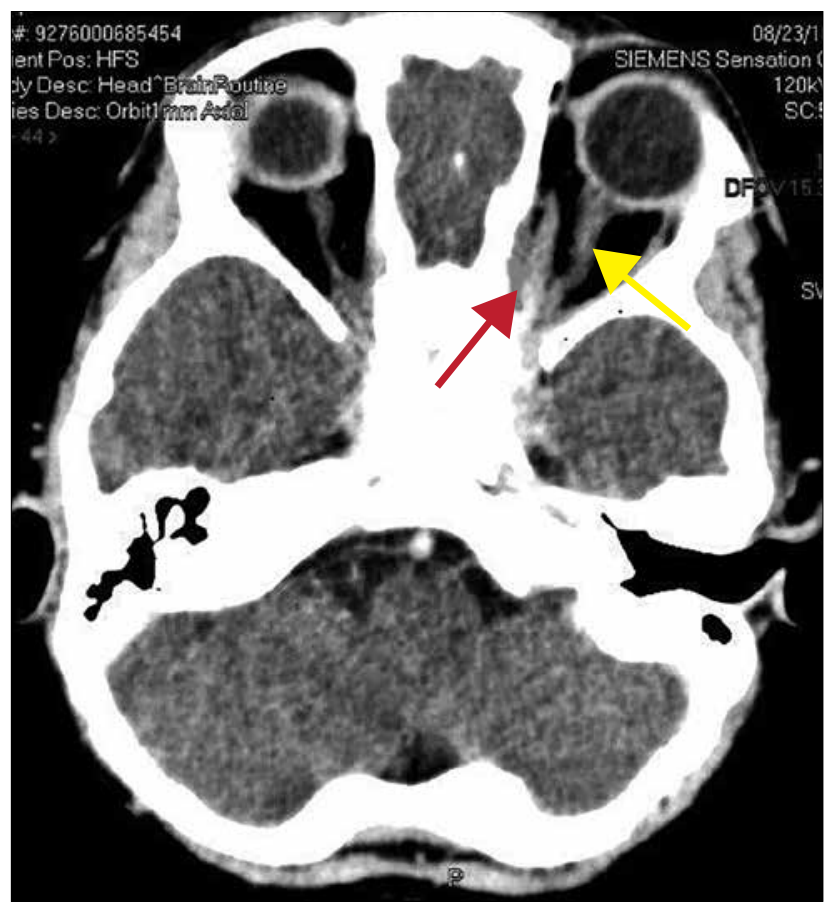

Fig. 1. Computed tomography scan showing medial fracture of the lamina papyracea with intraconal fat stranding (red arrow). The left optic nerve is thickened (yellow arrow).

- Brain. There were no intra- or extra-axial collections and no evidence of a haematoma formation or mass effect. The basal ganglia and posterior fossa structures were normal. No parenchymal or focal abnormality was seen. The basal cisterns and foramen magnum were patent. No fracture was seen on the calvarium.

- Conclusion. Left medial blow-out fracture through the anterior lamina papyracea with no entrapment of the extraocular muscles. Haematoma of the left extraocular muscles as described. Enlargement of the left optic nerve in keeping with orbital trauma.

\section{Discussion}

Orbital apex syndrome (OAS) has been described as a syndrome involving damage to the oculomotor nerve (CN3), trochlear nerve (CN4), ophthalmic branch of the trigeminal nerve (CN5) and abducens nerve (CN6) in association with optic nerve dysfunction. ${ }^{[1]}$ OAS consists of paralysis of all the extraocular muscles and a sensory deficit in the distribution of the first division of the trigeminal nerve, combined with an optic nerve lesion. ${ }^{[2]}$ The orbital apex is defined as the region between the posterior ethmoidal foramen and the openings of the optic canal and the superior orbital fissure. ${ }^{[3]}$ The apex of the orbital bone is the entry portal for all the nerves and vessels of the eye and the site of origin of all the extraocular muscles except the inferior oblique. ${ }^{[4]}$

OAS can be classified as having neoplastic, inflammatory, viral, fungal, traumatic or vascular causes. ${ }^{[5]}$ Although trauma is one of the main causes, ${ }^{[6]}$ reports of OAS associated with facial bone fractures are relatively rare. ${ }^{[7-10]}$ There are only 17 reported cases in the literature, with the locations of the fractures as follows: 7 medial wall, 7 lateral wall, 1 floor, 1 roof, 1 floor and roof. ${ }^{[6-10]}$ These fractures were probably due to a globe-to-wall contact mechanism. ${ }^{[12]}$ In the treatment of OAS associated with orbital fractures, the therapeutic targets are saving the globe, saving the third to sixth cranial nerves, and saving the optic nerve. ${ }^{[5]}$ The globe itself is highly vulnerable to permanent injury after an orbital fracture. Many complications from the trauma itself, such as hyphaema, lens dislocations or retinal detachments, as well as scleral rupture, may occur at the time of injury and contribute to permanent visual impairment. ${ }^{[5]}$ Early ophthalmological referral and management are imperative after any diagnosis of orbital fracture. ${ }^{[5]}$

Our patient was not referred early, and there was a 2-week delay before he was seen by an ophthalmologist. The nature of the fracture was not recognised at the first visit, and only a radiograph of the skull and facial bones was done. It was interpreted by the casualty officer, with no formal report by the radiologist, and the patient was sent home. He was next seen 9 days after the initial consultation, and by a different doctor. Only later, on suspicion that all was not well, was he referred to the eye clinic at the hospital. It was therefore 2 weeks after the initial injury that a CT scan of the brain and orbits was done. He was admitted to a tertiary hospital and given pulse steroid therapy over 3 days. This did not improve his condition, and there was no restoration of vision to the affected eye.

Non-operative intervention in OAS may consist of administration of high doses of corticosteroids to reduce microcirculatory spasm, oedema and nerve cell necrosis. ${ }^{[13]}$ The best outcomes occur with early diagnosis and early use of steroids, because irreversible ischaemic damage occurs within several hours. Treatment with steroids was unsuccessful in our patient because the time lapse between the injury and steroid administration was too great. ${ }^{[5]}$ In such cases, delayed and progressive loss of vision implies that a viable nerve is being compressed by blood and oedema. Early treatment may improve the prospects of recovery. ${ }^{[14]}$

\section{Conclusion}

This case report highlights the significance of prompt and appropriate radiological investigation of the patient with ocular injury, in the form of a CT scan at the time of first presentation. Importantly, if like ours the patient comes back to casualty repeatedly, the attending doctor should suspect that all is not well and there is an underlying pathology. A skull radiograph was not adequate in this case owing to the nature of the complaint, the age of the patient and the scanty history. Appropriate referral is important, as visual acuity was not assessed at the patient's first two visits, the IOP was not measured, and there was therefore no comment on what the condition of or vision in the eye was at the initial visit.

The child has been left with permanent loss of vision in the left eye, and it is quite possible that this could have been avoided with prompt intervention and referral.

Acknowledgements. The patient's parents gave formal written consent for the use of his CT images and medical case notes in this article. I also thank Prof. A Stulting for reviewing the article.

Author contributions. Sole author.

Funding. None.

Conflicts of interest. None.

1. Yeh S, Foroozan R. Orbital apex syndrome. Curr Opin Ophthalmol 2004:15(6):490-498. https://doi. org/10.1097/01.icu.0000144387.12739.9

2. Bray WH, Gianciacomo J, Ide CH. Orbital apex syndrome. Surv Ophthalmol 1987;32(2):136-140. https://doi.org/10.1016/0039-6257(87)90106-8

3. Ettl A, Zwrtek K, Daxer A, Salomonowitz E. Anatomy of the orbital apex and cavernous sinus on high resolution magnetic resonance images. Surv Ophthalmol 2000;44(4):303-323. https://doi.org/10.1016/ S0039-6257(99)00115-0

4. Shahid A, Ahmad I. Mucormycosis causing palatal necrosis and orbital apex syndrome. J Coll Physicians Surg Pak 2005;15(3):182-183.

Sugamata A. Orbital apex syndrome associated with fractures of the inferomedial orbital wall. Clin Ophthalmol 2013;7:475-478. https://doi.org/10.2147/OPTH.S42811

6. Peter NM, Peterson A. Orbital apex syndrome from blunt ocular trauma. Orbit 2010;29(1):42-44. https://doi.org/10.3109/01676830903190123 
7. Bernard A, Sadowsky D. Monocular blindness secondary to a non displaced molar fracture. Int J Oral Maxillofac Surg 1986;15(2):206-208. https://doi.org/10.1016/S0300-9785(86)80143-0

8. Acarturk S, Dalay C, Kivanc O, Varinli I. Orbital apex syndrome associated with fractures of the zygoma and orbital floor. Eur J Plast Surg 1993;16(2):67-69. https://doi.org/10.1007/BF00196434

9. Martello JY, Vasconez HC. Supraorbital roof fractures: A formidable entity with which to contend. Ann Plast Surg 1997;38(3):223-227. https://doi.org/10.1097/00000637-199703000-00006

10. Li Y, Wu W, Xiao Z, Peng A. Study on the treatment of traumatic orbital apex syndrome by nasal endoscopic surgery. Eur Arch Otorhinolaryngol 2011;268(3):341-349. https://doi.org/10.1007/s00405010-1409-6

11. Bater MC, Ramchandani PL, Ramchandani M, Flood TR. An orbital apex fracture resulting in multiple cranial neuropathies. Br J Oral Maxillofac Surg 2008;46(2):163-164. https://doi.org/10.1016/j. bjoms.2007.03.001
12. Sugamata A, Yoshizawa N. Clinical analysis of orbital blowout fractures caused by a globe to wall contact mechanism. J Plast Surg Hand Surg 2010;4(6):278-281. https://doi.org/10.3109/2000656X.2010.534614 13. Eo S, Kim JY, Azari K. Temporary orbital apex syndrome after repair of orbital wall fracture. Plast Reconstr Surg 2005;116(5):85e-89e. https://doi.org/10.1097/01.prs.0000182351.29929.7a

14. Lipkin IF, Woodson GE, Miller RH. Visual loss due to orbital fracture: The role of early reduction. Arch Otolaryngol Head Neck Surg 1987;113(1):81-83. https://doi.org/10.1001/archotol.1987.
A 01860010085021

Accepted 5 June 2017. 